\title{
ÉTICA CÍVICA: \\ INTEGRANDO LA EXPERIENCIA VIVIDA
}

\section{Verónica Gómez ${ }^{1}$, Paulina Royo ${ }^{2}$}

\author{
RESUMEN
}

El texto describe la experiencia de formular e impartir un curso de Ética ciudadana en un modelo de enseñanza-aprendizaje basado en competencias, en el contexto de una formación laica y humanista en la Universidad de Talca. Encuestas realizadas sugieren que este abordaje permitió a los estudiantes reforzar sus aprendizajes en el terreno de los valores cívicos y aplicar lo aprendido mucho más allá de la sala de clases.

Palabras clave: educación en valores, educación superior, ética aplicada

\section{CIVIC ETHICS: INTEGRATING EXPERIENCE}

\section{ABSTRACT}

This text describes the experience of designing and teaching a civic ethics course under a competence-based model in a non-religious higher education institution, the University of Talca. Surveys applied to the course participants suggest that this approach allowed students to further develop their abilities in the field of civic values and apply theses abilities well beyond the classroom.

Keywords: moral education, higher education, applied ethics

1 Vicerrectoría de Pregrado, Universidad de Talca. Contacto: vgomez@utalca.cl

2 Instituto de Estudios Humanísticos J.I. Molina, Universidad de Talca.

Contacto: proyo@utalca.cl 


\section{ÉTICA CÍVICA: \\ INTEGRANDO LA EXPERIENCIA VIVIDA}

\section{Introducción}

En 2005 la Universidad de Talca (UTAL) decidió reformar su currículum, adoptando el modelo de enseñanza-aprendizaje basado en competencias, el cual fue concebido en una doble perspectiva. En primer lugar, como "una combinación entre destrezas, habilidades y conocimientos necesarios para desempeñar una tarea específica" (U.S Department of Education, 2000, p. 1). Así, la competencia considera los conocimientos, habilidades y destrezas como medios que permiten alcanzar un fin: desempeñar efectivamente una tarea o actividad cumpliendo con los estándares requeridos en una determinada profesión, relevando el concepto de "aprendizaje basado en el desempeño" (González, Herrera y Zurita, 2009, pp. 21-31). En segundo término, enfatizando el "sabe[r] actuar de manera pertinente en un contexto particular, eligiendo y movilizando un equipamiento doble de recursos: recursos personales [...] y recursos de redes" (Le Boterf 2001:54), con un proceso de reflexión acerca de lo que se está haciendo.

En el caso específico de la UTAL se buscó potenciar, además, una educación para la ciudadanía responsable, que incluyera la necesidad de desarrollarse como persona y ser capaz de afrontar responsabilidades sociales. Se trataba de desarrollar las competencias genéricas o transversales que potenciaran el espíritu crítico y la formación de una ciudadanía activa, comprometida con los principios éticos de carácter universal.

Este último componente es, probablemente, el más complejo de incorporar en un modelo como este, debido a tres razones: a) si bien las universidades cuentan con una vasta experiencia en la entrega de conocimientos, la cuestión de las actitudes y disposiciones ha ido, frecuentemente, implícita en la visión y misión educativa de 
las instituciones de educación superior, lo cual significa que se trata de un elemento que no se traduce en un componente fácilmente identificable (y evaluable) dentro del currículo, excepto en aquellas instituciones asociadas a un proyecto religioso; b) al masificarse la educación superior chilena, resulta razonable suponer que cada vez más nos encontraremos con estudiantes cuya experiencia y opciones en cuanto a valores serán extremadamente heterogéneas ${ }^{3}$; c) la tercera y última razón es que el campo tradicionalmente a cargo de la formación del "saber ser" de las personas, es decir, de su ethos o personalidad moral, es hoy un terreno en disputa: los cambios sociales y las posibilidades tecnológicas implican enfrentar escenarios para los cuales no tenemos respuestas definidas por la reflexión ética. Giddens, Beck y Lash (1997) plantean que esta será una situación cada vez más frecuente en los años que vienen.

En este escenario, la Universidad de Talca decidió incorporar un curso de Ética ciudadana como parte de la malla de todas sus carreras. Originalmente denominado "Ética, Valoración y Sociedad" (EVS en lo futuro), dicha instancia de formación tuvo la misión de entregar herramientas para la reflexión ética y la toma de decisiones desde una visión humanista y laica. Esto significó pensar la enseñanza de la ética desde el punto de vista de su aplicación práctica, formulada en el lenguaje de las competencias: interesaba la enseñanza del modo de reflexionar propio de la ética moderna, basada en la aplicación de principios éticos universales. Ello, porque el modelo por competencias exige que se piense no solo en los contenidos que deben ofrecerse a los estudiantes, sino en la forma en que se espera que estos movilicen conocimientos y procedimientos en función de un objetivo concreto que se espera lograr como aprendizaje. Así, el currículo debe ser pensado como un conjunto de habilidades y procedimientos relacionados con el pensamiento ético, al cual se integran autores o corrientes filosóficas determinadas. Por ello, el enfoque basado en

3 A modo de ejemplo, de acuerdo con las cifras del Consejo Nacional de Educación en el año 2012 las universidades chilenas, privadas y miembros del Consejo de Rectores recibieron conjuntamente la matrícula aproximada 618.000 jóvenes. Cabe preguntarse, pues, si las fórmulas que ya conocemos -la difusión de un determinado proyecto ético-religioso, o bien, la adopción de un ethos implícito en la forma de operar de las instituciones de la educación superior- funcionarán en este nuevo escenario, marcado por la diversidad. 
competencias requiere que, junto con revisar los marcos conceptuales y analíticos de la ética y la filosofía moral modernas, los estudiantes incorporen explícitamente sus propias visiones de mundo y jerarquías de valores al proceso de aprendizaje.

Hechas estas distinciones preliminares, el presente artículo expone la experiencia obtenida entre los años 2007 y 2009 tras la ejecución del módulo de EVS, cuyo objetivo fue entregar a los y las estudiantes las herramientas necesarias para comprender las sociedades modernas, entendidas como espacios donde prima la pluralidad de jerarquías de valores y visiones de mundo y que, por ello, plantean la necesidad de establecer principios universales como base para la toma de decisiones comunes, los que se derivan de un acuerdo democrático alcanzado por la vía de la deliberación (Habermas, 1996).

Dichos principios a los que hacemos referencia se consideran cruciales para la convivencia democrática en aquellas sociedades en donde no existe una ética sustantiva compartida ${ }^{4}$.

\section{El módulo de "Ética, Valoración y Sociedad" (EVS)}

El módulo objeto del presente artículo se centró en la comprensión de las sociedades modernas como mundos sociales racionalizados (en el sentido weberiano del término) donde se reconocen los derechos de las personas basándose en su condición de seres humanos y no en la pertenencia a una comunidad de valores determinada. Con ello, se quiso enfatizar en la importancia de las ideas de diálogo y tolerancia, así como también en la del respeto a concepciones distintas de vida buena.

Así pues, sobre esta base, el módulo de EVS ofreció insumos para la reflexión ética desde el punto de vista de las éticas procedimentales basadas en principios universales. Su objetivo último radicó en que los

4 No es este el lugar para volver al debate entre liberales y "comunitaristas" acerca de las posibilidades de distinguir, aunque sea analíticamente, entre el sujeto ético y la comunidad de valores de la cual forma parte, debate que excede el propósito de este trabajo. 
y las estudiantes fueran capaces de fundamentar sus opciones valóricas personales en dichos principios éticos, con lo cual podrían propender hacia la construcción de acuerdos democráticos en el futuro. Esta competencia de mayor complejidad fue formulada como sigue:

\begin{tabular}{|l|l|}
\hline $\begin{array}{l}\text { COMPETENCIA: Fundamentar las opciones valóricas personales en principios éticos para la construcción } \\
\text { de acuerdos democráticos. }\end{array}$ \\
\hline Subcompetencia 1 & $\begin{array}{l}\text { Identifica los fundamentos éticos de la limitación de su propia libertad en } \\
\text { relación con las libertades de otras personas en su comunidad. }\end{array}$ \\
\hline Subcompetencia 2 & $\begin{array}{l}\text { Identifica los fundamentos éticos de la existencia de cursos de acción diversos } \\
\text { o contradictorios, siempre que estos se ajusten a mínimos éticos previamente } \\
\text { consensuados por la colectividad (derechos/deberes). }\end{array}$ \\
\hline Subcompetencia 3 & $\begin{array}{l}\text { Fundamentar sus opciones valóricas personales en principios universales para } \\
\text { la construcción de acuerdos democráticos. }\end{array}$ \\
\hline
\end{tabular}

A partir de ello, se definió un conjunto de contenidos y estrategias metodológicas que se consideraron apropiadas para los propósitos del módulo.

Ahora bien, tradicionalmente las cuestiones relacionadas con filosofía, ética y derechos humanos han sido asociadas a la adquisición de un discurso teórico y no necesariamente a la movilización de recursos personales de los y las estudiantes -creencias, experiencias, opciones valóricas- en el proceso de aprendizaje. Sin embargo, el enfoque basado en competencias es altamente compatible con metodologías de trabajo que involucran activamente a los y las estudiantes, tales como análisis de material audiovisual, casos ficticios basados en información real y sesiones de deliberación. Por este motivo, se optó por formas de trabajo en sala y una evaluación que acercara la reflexión ética hacia la experiencia cotidiana de los y las estudiantes, de modo tal que la misma fuera reexaminada a la luz de los marcos analíticos proporcionados por la ética.

Lo anterior implicó reconocer la importancia de desarrollar en los y las estudiantes la capacidad de evaluar situaciones que plantearan más de una opción éticamente justificable y otorgarles un sentido valórico, operación que frecuentemente demanda una "negociación" -desde el punto de vista del sentido- respecto de las propias opciones personales en el terreno de los valores (Light, Cox y Calkins, 2009, p. 22). En las páginas siguientes examinaremos dos de estas formas de trabajo: los análisis de caso y las sesiones de deliberación. 


\section{Análisis de caso en el módulo de EVS}

Argandoña (1999) señala que una objeción frecuente hacia la enseñanza de la ética es que: "Los criterios y valores morales de los alumnos están ya formados (o deformados) y no se puede pretender cambiarlos en unas cuantas clases". Coincidimos con Argandoña en que la réplica más acertada a dicha objeción es que el proceso de aprendizaje moral está siempre abierto: los valores se van consolidando o deformando y no hay por qué negar a los alumnos y alumnas la oportunidad de mejorar su formación ética (Piper, Gentile y Parks, 1993), particularmente cuando el entorno lo demanda.

Desde esta perspectiva podemos señalar que la unidad moral de la persona exige una formación integral que abarque todas las facetas de la vida, y la creciente demanda hacia las universidades por profesionales capaces de enfrentar situaciones complejas convierte a este aprendizaje en una tarea ineludible. Este mismo hecho (que se trate de personas adultas con experiencias y valores definidos) representa una oportunidad cuando el objetivo no es "imprimir" una cierta visión de mundo en nuestros estudiantes, sino fortalecer su capacidad de autodescubrimiento en el plano ético. Esto es condición indispensable para la autonomía, entendida como la capacidad de "agenciar" la vida (Sen, 2000), aprendiendo a ejercer de manera más reflexiva la libertad individual, desde las propias experiencias y convicciones, cuestionando críticamente presiones externas o planteamientos heterónomos.

Mediante este modelo, pues, se busca ampliar las facultades para que los y las estudiantes sean sujetos protagonistas de las decisiones que tomen en sus vidas. Las personalidades maduras desde el punto de vista axiológico suponen este "señorío de sí" que integra aspectos tales como "autodisciplina, fortaleza, constancia, autocontrol y autodominio” (Díaz, 2001, p. 135). Paradójicamente, también se necesita una conciencia autónoma para "renunciar a una postura personal cuando se reconocen mejores razones en el adversario" (García y Puig 2008).

En los dilemas éticos - casos en los cuales hay más de una opción compatible con valores socialmente compartidos- el juicio 
ético requiere la capacidad de anticipar los efectos morales y prácticos de las acciones y ponderar los valores involucrados. Por ello, creemos que representan un buen instrumento para involucrar a los y las estudiantes en la reflexión ética.

Desde el punto de vista metodológico y en el contexto del módulo de EVS un caso fue entendido como una relación escrita que describía una situación acaecida en la vida de una persona, familia, grupo o empresa. Su aplicación como estrategia o técnica de aprendizaje buscaba entrenar a los estudiantes en la elaboración de soluciones válidas para los posibles problemas de carácter complejo que se presentarán en la realidad futura. Según la Dirección de Investigación y Desarrollo Educativo del Instituto Tecnológico y de Estudios Superiores de Monterrey, lo que hace particularmente importante a un caso es lo siguiente:

"El caso no proporciona soluciones, sino datos concretos para reflexionar, analizar y discutir las posibles salidas que se pueden encontrar a cierto problema. No ofrece las soluciones al estudiante, sino que le entrena para generarlas. Le lleva a pensar y a contrastar sus conclusiones con las conclusiones de otros, a aceptarlas y expresar las propias sugerencias [...] Al llevar al alumno a la generación de alternativas de solución, le permite desarrollar la habilidad creativa, la capacidad de innovación y representa un recurso para conectar la teoría con la práctica real" (s.d. itálicas nuestras).

En el módulo de EVS, los casos fueron utilizados para examinar, en un primer momento, la idea de libertad individual como un espacio delimitado por un derecho igual para todos. Dentro de su diversidad, el caso-ejemplo parecía ser especialmente adecuado para este propósito. En esta modalidad se discutieron casos de situaciones paradigmáticas (llamados casos cerrados) previamente analizadas y resueltas por especialistas, con el fin de presentar el conocimiento y la comprensión de los procesos de análisis llevados a cabo, así como los recursos teóricos utilizados para comprender la situación y llegar a una decisión razonada. Son, por así llamarlos, casos modelo que podían examinarse en sala. 
Posteriormente, en oposición a los casos cerrados también se recurrió a los casos abiertos, los cuales constituyeron una importante herramienta para identificar los fundamentos éticos de la existencia de cursos de acción diversos frente a un mismo problema -diversidad que está limitada por los principios de carácter universal-. La multiplicidad de cursos de acción no se convertía así en subjetividad (un prejuicio recurrente de los y las estudiantes frente a la ética), sino en intersubjetividad, es decir, en el resultado de opciones colectivas y conscientes.

Este segundo tipo de caso enfrentaba al estudiante a una situación no resuelta previamente, puesto que buscaba entrenarlos en su resolución, lo que si bien requería de habilidades teóricas también exigía que se atendiera la singularidad y la complejidad de contextos específicos.

Ahora bien, a diferencia de la deontología más prescriptiva, los principios éticos de carácter universal requieren de una atención particular respecto del contexto y las condiciones específicas de la situación a la cual se aplican. Así, durante el módulo se subrayaba el respeto por las opciones valóricas personales, por lo cual en las situaciones presentadas no se daba la respuesta correcta, sino que se generaba una discusión en la cual los y las estudiantes llegaban a sus propias conclusiones, guiados por el docente, quien debía estar abierto a soluciones diversas.

Por otra parte, al utilizar casos de este segundo tipo (casos abiertos) en el módulo de EVS se pedía a los y las estudiantes que realizaran cuatro operaciones básicas:

a) Identificar la controversia ética, es decir, señalar por qué el caso planteado presenta un dilema ético.

b) Identificar los principales actores involucrados, así como los respectivos argumentos.

c) Examinar por lo menos dos cursos de acción posibles y sus consecuencias frente al dilema identificado, con lo cual se esperaba que los y las estudiantes ponderaran distintas posibilidades.

d) Seleccionar, a partir de las opciones identificadas en el punto precedente, un curso de acción como opción personal preferida, 
fundamentando la decisión. Para ello, el estudiante debía aplicar, por lo menos, un principio ético y dos conceptos trabajados durante el módulo.

Respecto de esta última operación básica, ella requería que el estudiantado se comprometiera con un curso de acción o decisión, según su jerarquía personal de valores, así como también demandó capacidades analíticas para el estudio y decisión acerca de problemas morales, pues debían ofrecerse fundamentos para dicha decisión.

De esta forma se buscaba, específicamente, que el estudiante introdujera su propia experiencia y valores en el ejercicio, reexaminándolos a la luz de los marcos analíticos revisados en el módulo. Asimismo, ofrecer fundamentación requería contrastar las propias opciones personales con parámetros predefinidos, lo cual es, con frecuencia, uno de los puntos que presenta más dificultad para los y las estudiantes.

Por otra parte, las operaciones señaladas arriba constituyeron también los ítems considerados para una de las evaluaciones centrales del módulo. En efecto, el eje de la evaluación estuvo en el proceso de análisis y la fundamentación de la decisión, para lo cual se definieron criterios que fueran conocidos por el estudiantado desde el inicio de la experiencia de formación. Por ejemplo, en el punto referido a la opción personal fundamentada se evaluó la pertinencia de los principios y conceptos seleccionados para justificar el curso de acción escogido, así como también la utilización rigurosa de los marcos analíticos y conceptuales vistos en el transcurso del módulo. La rúbrica ${ }^{5}$ para este punto se muestra en la siguiente tabla:

5 La rúbrica corresponde a la matriz de valoración que se utiliza para calificar el desempeño de los estudiantes en un modelo educativo basado en competencias. 


\begin{tabular}{|c|c|c|c|c|}
\hline No logrado & Recuperable & Básico & Habilitado & Destacado \\
\hline $\begin{array}{l}\text { Los conceptos/ } \\
\text { principios } \\
\text { utilizados para } \\
\text { fundamentar la } \\
\text { opción por un } \\
\text { curso de acción no } \\
\text { son pertinentes, } \\
\text { no se los define } \\
\text { o la definición es } \\
\text { incorrecta. }\end{array}$ & $\begin{array}{l}\text { Los conceptos/ } \\
\text { principios } \\
\text { utilizados son } \\
\text { pertinentes, pero } \\
\text { las definiciones } \\
\text { requieren ser } \\
\text { precisadas. No } \\
\text { se los relaciona } \\
\text { explícita y } \\
\text { lógicamente con } \\
\text { el caso. }\end{array}$ & $\begin{array}{l}\text { Los conceptos/ } \\
\text { principios } \\
\text { utilizados son } \\
\text { pertinentes y } \\
\text { están definidos de } \\
\text { manera adecuada, } \\
\text { pero no se los } \\
\text { relacionan explícita } \\
\text { y lógicamente con } \\
\text { el caso. }\end{array}$ & $\begin{array}{l}\text { Los conceptos/ } \\
\text { principios } \\
\text { utilizados son } \\
\text { pertinentes y } \\
\text { están definidos } \\
\text { de manera } \\
\text { adecuada. Se los } \\
\text { ha relacionado } \\
\text { explícita y } \\
\text { lógicamente con } \\
\text { el caso. }\end{array}$ & $\begin{array}{l}\text { Los conceptos/ } \\
\text { principios } \\
\text { utilizados son } \\
\text { pertinentes, están } \\
\text { definidos de } \\
\text { manera adecuada } \\
\text { y relacionados } \\
\text { explícita y } \\
\text { lógicamente con } \\
\text { el caso y con otros } \\
\text { casos del mismo } \\
\text { tipo. }\end{array}$ \\
\hline
\end{tabular}

Como puede observarse en la tabla, la evaluación consideró la coherencia del análisis, más que el componente sustantivo de la decisión. Así, dado que ninguna opción no susceptible de ser justificada utilizando un principio universal podía ser aceptada como resultado del análisis ético, se excluyeron aquellas decisiones que no podían ser defendidas públicamente, contribuyendo de esta forma a reducir la idea del relativismo en el ámbito de las decisiones éticas.

\section{Decidiendo con otros: la deliberación aplicada al módulo de EVS}

Si bien el análisis de caso puede utilizarse con grupos o individualmente, en la experiencia que se está describiendo (el módulo de EVS) se relevó la capacidad de los y las estudiantes para sistematizar y examinar, críticamente, las intuiciones éticas con las que llegaban al curso. Por ello, la competencia final (y la más compleja) del módulo añadía a la capacidad de decidir autónomamente el requisito de movilizar dicha capacidad para construir acuerdos con los otros. El origen de esta competencia, de cuño habermasiano ${ }^{6}$, posibilitó que los estudiantes orientaran el proceso decisorio hacia el logro de un consenso que pudiera ser aceptado por todos los actores afectados por la decisión. En síntesis, se trataba de hacer el ejercicio empático de considerar varios puntos de vista y "ponerse en los zapatos del otro" para asegurar la racionalidad ética de la decisión.

6 De Jürgen Habermas, filósofo y sociólogo alemán, ampliamente conocido por sus trabajos en filosofía práctica (ética, filosofía política y del derecho). 
La metodología descrita, llamada método deliberativo o deliberación, era aplicable al módulo de EVS precisamente porque en ética se delibera por la existencia de diversos grados de certeza de los conocimientos. En este caso particular, el principal desafío fue producir un vínculo significativo y consciente entre la existencia de la doxa que portaba el estudiantado y el fortalecimiento de la episteme que podría adquirir, utilizando un tipo de razonamiento propio de la racionalidad práctica.

En el aula este ejercicio dialéctico les permitió tomar decisiones producto de valores que se habían reformulado, complementado y revalidado a través del diálogo con los otros. Así, durante esta experiencia de formación invitamos a los y las estudiantes a razonar y poner en juicio la legitimidad de ciertos principios, cuyos criterios de validez se relacionaban con la universalidad, imparcialidad y solidaridad que deben estar necesariamente implicados en los principios éticos que se proponen.

Estamos ciertos de que el diálogo es el único modo en el que las personas pueden averiguar si sus necesidades pueden defenderse intersubjetivamente como exigencias, o bien deben permanecer en el terreno de lo subjetivo. Si una necesidad posee base argumentativa suficiente como para plantearse como exigencia (objetiva), es que puede pretender con todo derecho convertirse en fundamento para una norma moral en la vida pública. Este enfoque se basa en la tradición de ética comunicativa actualizada por Karl Otto Apel y Jürgen Habermas.

El método deliberativo o simplemente la deliberación lo entendemos, además, como un "proceso de ponderación de los factores que intervienen en un acto o situación concreta, a fin de buscar su solución óptima o, cuando esto no es posible, la menos lesiva [...] Se delibera sobre lo que permite diferentes cursos de acción, en orden a buscar el más adecuado [...]" (Gracia, 2001, p. 22).

Como vemos, los presupuestos básicos del método deliberativo o deliberación se relacionan con el imperativo de decidir en condiciones de incertidumbre, permitiendo la participación 
argumentativa de todos los actores potencialmente afectados. Con esta metodología, pues, se intenta superar la fundamentación basada en la tradición, la autoridad, o bien, las expectativas externas, así como también tiene el propósito de potenciar una perspectiva racional y reflexiva que permita recopilar antecedentes y aplicar criterios de evaluación ajustados a situaciones de la vida real.

4. Resultados de la experiencia en el módulo de EVS: la visión de los estudiantes

Para tener una visión del resultado de esta experiencia desde el punto de vista de los y las estudiantes, en 2009 se aplicó una encuesta anónima para recopilar la percepción de los cerca de mil estudiantes que cursaron el módulo. Dentro de la batería de preguntas, se incluyó una abierta en la cual se les daba la oportunidad de señalar, en su propio lenguaje, lo que habían aprendido. Las respuestas fueron sometidas a un proceso de categorización cuyos resultados se presentan a continuación:

Gráfico 1: ¿Cuáles consideras que fueron tus principales aprendizajes en este módulo? (Pregunta abierta, N= 555). Módulo de EVS, 2009.

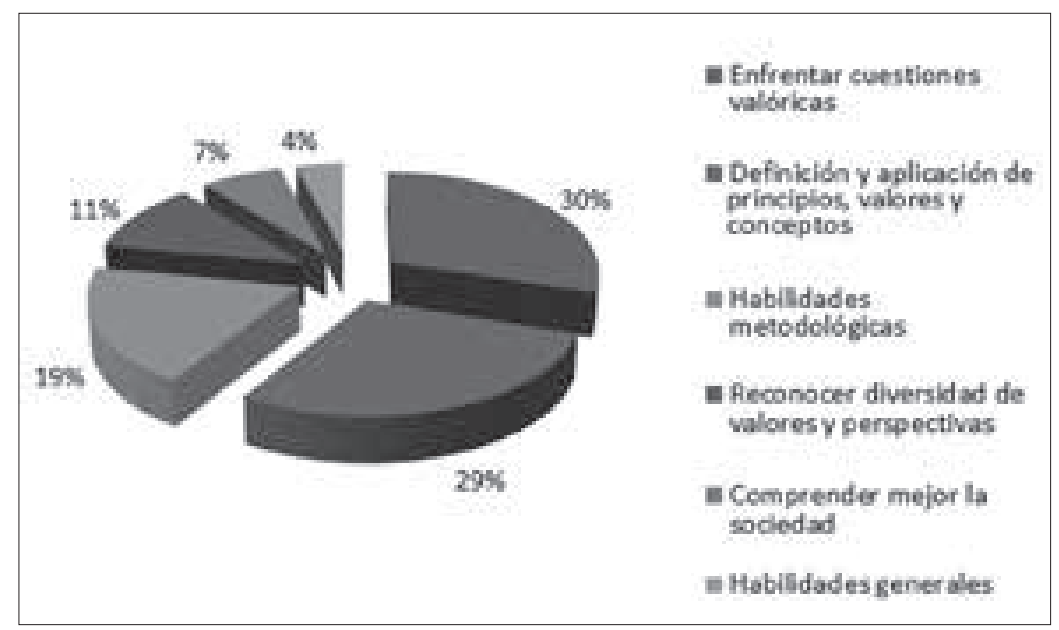

Tal como se aprecia en la gráfica, un 30\% de los y las estudiantes señaló como principal aprendizaje el "enfrentar cuestiones valóricas", 
categoría que incluía la forma de abordar dilemas, ponderar aspectos éticos de un problema y sus implicaciones y resolver o enfrentar conflictos de valores de manera tolerante, desde el punto de vista del cambio que los propios estudiantes percibían en su modo de enfrentar cuestiones valóricas. Por su parte, un porcentaje similar (29\%) señaló como principal aprendizaje la definición y aplicación de principios, valores y conceptos, en suma, aprendizajes identificados en el plano de la teoría ética, tales como la distinción entre ética y moral, problemas base de la reflexión ética, formulación de principios y utilización de un lenguaje especializado. Un 19\%, en tanto, destacó las habilidades metodológicas adquiridas (aprendizajes en el plano de metodologías propias del análisis ético, así como procedimientos propios de dicho análisis, tales como pasos del análisis, contrastación de juicios, modelo de la conciencia ética, método deliberativo). El 11\% de las respuestas señalaron el reconocer la diversidad de valores y perspectivas como algo positivo, mientras que un 7\% identificó aprendizajes desde el punto de vista de comprender situaciones sociales que tienen un aspecto ético importante. Finalmente, un 4\% destacó aprendizajes vinculados a habilidades no específicas del análisis ético, agrupadas bajo la categoría de "habilidades generales".

En la misma encuesta se preguntó a los y las estudiantes dónde podrían aplicar lo aprendido. El ítem fue cerrado, de opción múltiple, y es importante hacer notar que un 80\% de los estudiantes reconoció que podrían aplicar lo aprendido en contextos distintos de la sala de clases: nadie marcó la opción "ninguno". 
Gráfico 2: ¿En qué contexto(s) consideras que podrías aplicar lo aprendido? (Porcentaje que marcó la opción. N=675). Módulo de EVS, 2009.

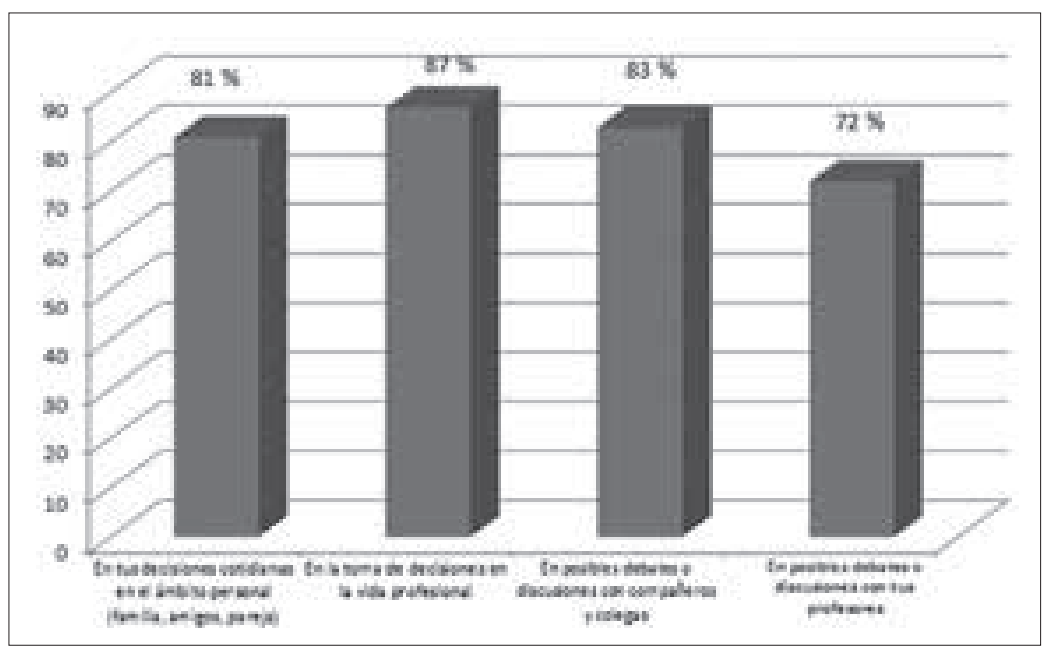

Esto sugiere que los y las estudiantes vislumbraron formas de transferir las habilidades y conceptos aprendidos durante el módulo a otros contextos de su vida, en lugar de considerar que la ética es una materia que debe "quedarse en el cuaderno".

\section{Principales dificultades tras la experiencia del módulo de EVS}

Pese a los auspiciosos resultados evidenciados por la encuesta aplicada, la experiencia de formación que nos ocupa no ha estado exenta de dificultades. Entre las problemáticas más destacables podemos mencionar la resistencia del estudiantado a superar la tendencia de situar las decisiones éticas en el plano de lo puramente subjetivo. Efectivamente, en una universidad de carácter marcadamente técnicocientífico, como es el caso de la Universidad de Talca, no tener respuestas unívocas le resta validez a la ética, frente a la pretensión de objetividad de la ciencia. Específicamente, en las etapas iniciales del módulo, esta resistencia se expresaba con frecuencia en la demanda por "recetas" o respuestas "correctas" únicas y, cuando estas no llegaban, se asumía (a veces con frustración) que a la larga "todo es subjetivo". 
El equipo docente a cargo del módulo enfrentó esta dificultad sistematizando el trabajo de clases y extraula mediante instructivos que describían las etapas de análisis ético y destacando la rigurosidad propia de la racionalidad práctica, distinta de la racionalidad instrumental de la ciencia, pero destacando también el margen correspondiente a la libertad humana.

Como medida complementaria, dentro de los contenidos del módulo se especificó la distinción entre diferentes tipos de racionalidad, pero no por ello menos rigurosas. Contar con estos instructivos y con pautas de evaluación explícitas que enfatizaban el procedimiento utilizado para la toma de decisiones, más que el componente sustantivo de las mismas, contribuyó significativamente a debilitar la mencionada resistencia, así como también otra asociada, esta vez proveniente del profesorado: el temor de que se intentara ideologizar la enseñanza de la ética, privilegiando ciertas visiones de mundo por sobre otras.

En este segundo escenario, el proceso para revertir las percepciones negativas se dio por aproximaciones sucesivas: cada vez que surgía un cuestionamiento recurrente del estudiantado, los docentes nos vimos motivados a hacer explícito el recorrido metodológico (y de razonamiento) que explicara la diferencia existente entre una decisión basada en referentes exclusivamente personales y otra, que es el resultado de la reflexión sistemática y del ejercicio empático de asumir la posición de un otro hipotético.

\section{Conclusiones}

La innovación representada por el cambio de paradigma de una deontología teórica y prescriptiva a un modelo de ética aplicada es un desafío significativo, tanto para estudiantes como para los docentes, dado que al potenciar la capacidad de los primeros para tomar decisiones autónomamente, se multiplican las oportunidades de problematizar las cuestiones propias de la ética.

Lo anterior, por una parte, representa una instancia valiosa de aprendizaje para todos los involucrados que exige un compromiso 
mayor con el trabajo en sala y los procesos de evaluación; y, por otra, revela que los estudiantes están poco habituados a enfrentar situaciones de aprendizaje abiertas, en las cuales ellos y ellas deban organizar la información y analizarla desde su propia jerarquía de valores, utilizando las herramientas de una metodología ética sistemática y fundada. Ello se ve reflejado, por ejemplo, en la transición que realizan los estudiantes desde una preferencia por decisiones orientadas por reglas, hacia una racionalidad práctica más orientada por principios universales.

Los resultados de la encuesta aplicada a los y las estudiantes participantes del módulo "Ética, Valoración y Sociedad", impartido en la Universidad de Talca, también señalan que, al tratarse de una ética aplicada que demanda la movilización de los recursos personales, como resultado facilita que ellos transfieran sus aprendizajes $-\mathrm{O}$ al menos puedan imaginarse cómo hacerlo-a otros contextos personales y profesionales. A través del análisis ético y del diálogo, los estudiantes tuvieron la posibilidad de reexaminar sus convicciones y valores personales de manera activa en un contexto de aprendizaje formal.

Este desafío metodológico fue asumido con la esperanza de contribuir con la formación de hombres y mujeres capaces de ejercer una ciudadanía más responsable. Al fortalecer sus capacidades de autonomía y ejercitarlas en su libertad personal, nos asistió la profunda convicción de que ellos y ellas podrían contribuir a la consolidación de los valores que fundamentan la vida democrática.

\section{Referencias bibliográficas}

Argandoña, A. (1999). La enseñanza de la ética por el método del caso. En J. R. Flecha (Ed.), Europa: ¿mercado o comunidad? De la Escuela de Salamanca a la Europa del futuro (pp. 22 a 47). Salamanca: Publicaciones Universidad Pontífica.

Díaz, C. (2001). Las claves de los valores. Madrid: Ediciones Internacionales Universitarias, S.A.

Dirección de Investigación y Desarrollo Educativo del Instituto Tecnológico y de Estudios Superiores de Monterrey (s.d.). Las estrategias y técnicas didácticas en el rediseño. El estudio de casos como técnica didáctica. 
Disponible en http://tecnologiaedu.us.es/dipro2/images/stories/M10/ PDF/pdf_11/page_01.htm

García, M. y Puig, J. (2008). Las siete competencias básicas para educar en valores. Barcelona: Graó.

Giddens, A., Beck, U. y Lash, S. (1997). Modernización reflexiva. Madrid: Alianza.

Gracia, D. (2001). La deliberación moral: el método de la ética clínica. Medicina Clínica, 10 (117), pp. 18-23.

González, E., Herrera, R. y Zurita, R. (2009). Formación Basada en Competencias: Desafíos y Oportunidades en Diseño Curricular Basado en Competencias y Aseguramiento de la Calidad en la Educación Superior. Santiago de Chile: Cinda.

Habermas, J. (1996). Between facts and norms. Towards a discursive theory of democracy. Cambridge (Mass.): The MIT Press.

Le Boterf, G. (2001). Ingeniería de competencias. París: Editorial Gestión 2000.

Light, G., Cox, R. y Calkins S. (2009). Learning and teaching in higher education. The reflective professional. Londres: SAGE.

Piper, T., Gentile, M. y Parks, S. (1993) Can ethics be taught? Boston: Harvard Business School.

U.S Department of Education, National Center for Education Statistics (2000). Defining and assessing learning: exploring competency-based initiatives. Washington, D.C.: U.S. Department of Education, National Center for Education Statistic.

Recibido: 31/08/2012

Aceptado: 07/11/2012 